\title{
Investigation of High Efficiency SIMPLE Algorithm Based on Single Staggered Grid for Sewage Treatment System
}

\author{
Guan-Nan LIU ${ }^{1,2, a}$, Mao-Chang DUAN ${ }^{3, b}$, Feng GAO ${ }^{1,2, c}$, Yu-Gui YANG ${ }^{1,2, d}$
}

${ }^{1}$ School of mechanics \& civil engineering, China University of Mining \& Technology, Xuzhou, Jiangsu, 221116, China

${ }^{2}$ State Key Laboratory for Geomechanics \& Deep Underground Engineering, China University of Mining \& Technology, Xuzhou, 221008, China

\author{
${ }^{3}$ School of Aeronautic Science and Engineering, Beihang university,Beijing, 100191, China \\ aguannanliu@126.com, bssnn521@126.com, ${ }^{c}$ iflove0922@126.com, dyouxiang_liu@126.com
}

Keywords: Sewage Treatment, Staggered Grid, Flow Field, Collocated Grid.

\begin{abstract}
Aiming at the problem of wastewater treatment system, the Navier-Stokes equations of incompressible flow were calculated by one-direction staggered grid method. The reason and inhibiting method of the unreasonable pressure field was dicussed in the article. The expressions of correction equations of speed in different directions were derived. As an example, the speed field and temperature field of liquid flow at right angles were simulated. The results showed that the maximum temperature difference between both ends of the model is about $50^{\circ} \mathrm{C}$, and the minimum temperature difference between the both ends is about $30^{\circ} \mathrm{C}$ at $\mathrm{y}=0.375 \mathrm{~m}$. The lowest temperature is at the exit of the flow field. The results were compared with the staggered grid results, which verifies the calculation method proposed in this article is reasonable.
\end{abstract}

\section{Introduction}

With the complicated and refined of sewage treatment system, to build accurate and efficient numerical method is a problem urgently to be solved in recent years. Fluid flows through the orthogonal plane problem widely exists in the field of sewage treatment, which has important engineering significance. SIMPLE algorithm and the improved scheme is widely used in incompressible flow of the numerical solution of the Navier - Stokes equations. In order to solve the flow field in the oscillating pressure field, staggered grid system were usually chosen, in which the pressure and velocity components are stored on different grid. Staggered grid system adapt to complicated boundary problem weakly, and brings complicated calculation process in curvilinear coordinate system [1-3]. At present, some researchers using non staggered grid to eliminate the unreasonable pressure field has made some progress, but the application of this method need to be further verified [4-6]. Single direction staggered grid system is presented in this article. Non-mainstream direction speed variable and scalars stored in the same set of grid to improve the efficiency of numerical solution under the premise of ensure accuracy. From the previous studies, three important observations emerged:

1. Staggered grid algorithm has higher precision but huge calculation amount(especially for three-dimension problem) and weak grid adaptability, because there are more than one set of grid system.

2. collocated grid algorithm has small amount of calculation but lower precision, because of the momentum interpolation.

3. Half staggered grid algorithm also has lower precision, because of the momentum interpolation.

Different from previous studies, this research proposed a single direction staggered grid algorithm. The velocity of the main flow direction are calculated by staggered grid algorithm, but other velocities are calculated by collocated grid algorithm. The feasibility of the method were proved by comparing with the results of staggered grid algorithm. 


\section{Basic Equations}

The mass continuity equation,momentum equation,energy equation and composition equation could be expressed as common version [7]:

$$
\frac{\partial(\rho \phi)}{\partial t}+\operatorname{div}(\rho \vec{u} \phi)=\operatorname{div}(\Gamma \operatorname{grad} \phi)+S
$$

Where $\phi$ is a universal variable,including $u, v, w, T$ et al; $\Gamma$ is the general diffusion coefficient; $S$ is the general source term, and $\phi, \Gamma, S$ have different forms.

Standard $k-\varepsilon$ model is mainly based on turbulent kinetic energy and turbulent diffusivity. The flow field is assumed to be completely turbulent in Standard $k-\varepsilon$ model, and the molecular viscosity is ignored [8-9].

$$
\begin{aligned}
& \frac{\partial}{\partial t}(\rho k)+\frac{\partial}{\partial x_{i}}\left(\rho k u_{i}\right)=\frac{\partial}{\partial x_{j}}\left[\left(\mu+\frac{\mu_{t}}{\sigma_{k}}\right) \frac{\partial k}{\partial x_{j}}\right]+G_{k}+G_{b}-\rho \varepsilon-Y_{M}+S_{k} \\
& \frac{\partial}{\partial t}(\rho \varepsilon)+\frac{\partial}{\partial x_{i}}\left(\rho \varepsilon u_{i}\right)=\frac{\partial}{\partial x_{j}}\left[\left(\mu+\frac{\mu_{t}}{\sigma_{\varepsilon}}\right) \frac{\partial \varepsilon}{\partial x_{j}}\right]+C_{1 \varepsilon} \frac{\varepsilon}{k}\left(G_{k}+C_{3 \varepsilon} G_{b}\right)-C_{2 \varepsilon} \rho \frac{\varepsilon^{2}}{k}+S_{\varepsilon}
\end{aligned}
$$

Where $G_{k}, G_{b}$ represent the engender term of turbulent kinetic energy by average velocity gradient and buoyancy separately, $Y_{M}$ represents the contribution by pulsation dilation in compressible turbulent flow, $G_{1 \varepsilon}, G_{2 \varepsilon}$ and $G_{3 \varepsilon}$ are expirical constants, $\sigma_{k}$ and $\sigma_{\varepsilon}$ are the Prandtl numbers associated with turbulent kinetic energy and turbulent dissipation rate, $S_{k}$ and $S_{\varepsilon}$ are source terms.

\section{The Numerical Solution Method}

In single staggered grid method, scalar and v component are stored in the same set of grid system, and $\mathrm{u}$ component stored in half the length of the grid with the master control body staggered grid system [10-12], as shown in Fig. 1.

- $\mathrm{N}$

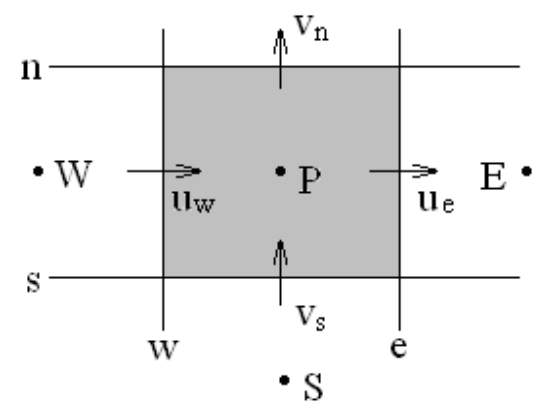

(a) Main Control Volume v Control Volume

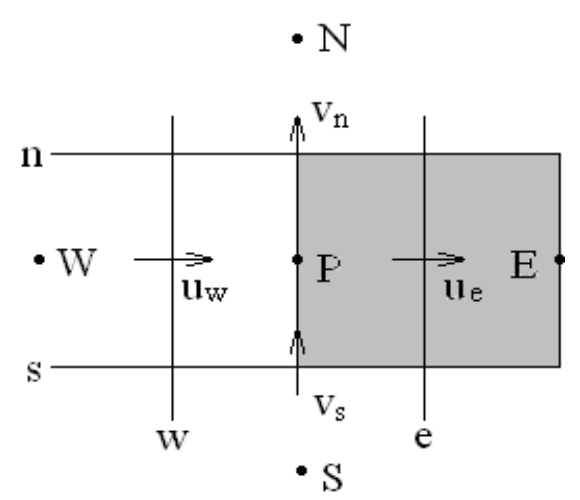

(b) u Control Volume

Fig.1 Sketch Map of Stagger Grid

Assume the initial stress field of speculation is $p^{*}$, For the $\mathrm{v}$ direction(pipeline radial), momentum difference method was adopted: 


$$
v_{I, j+1}=\frac{\sum a_{n b} v_{n b}^{*}+b_{I, j+1}}{a_{I, j+1}}-\frac{1}{2}\left(\frac{A_{I, J+1}}{a_{I, J+1}}+\frac{A_{I, J}}{a_{I, J}}\right)\left(p_{I, J+1}^{*}-p_{I, J}^{*}\right)
$$

SIMPLE algorithm was used to omitted the correction value of neighbor points:

$$
v_{I, j+1}^{\prime}=\frac{1}{2}\left(\frac{A_{I, J+1}}{a_{I, J+1}}+\frac{A_{I, J}}{a_{I, J}}\right)\left(p_{I, J}^{\prime}-p_{I, J+1}^{\prime}\right)
$$

According to the definition of the speed correction, we can calculate the $\mathrm{v}$ as follows:

$$
v_{I, j+1}=v_{I, j+1}^{*}+d_{I, j+1}\left(p_{I, J}^{\prime}-p_{I, J+1}^{\prime}\right)
$$

Define the revised pressure $p^{\prime}$ to the difference between the correct pressure field $p$ and the speculated pressure field $p^{*}$, The staggered grid discrete format was used in the $\mathrm{u}$ direction. The discrete format of the momentum equations could be solved by the pressure field.

$$
a_{i, J} u_{i, J}^{\prime}=\Sigma a_{n b} u_{n b}^{\prime}+\left(p_{I-1, J}^{\prime}-p_{I, J}^{\prime}\right) A_{i, J}
$$

In order to simplify the solving process, we introduce the following approximate calculation: $\sum a_{n b} u_{n b}^{\prime}$ which related the revised speed in the equation is omitted. So:

$$
u_{i, J}^{\prime}=\left(p_{I-1, J}^{\prime}-p_{I, J}^{\prime}\right) d_{i, J}
$$

By the definition of the revised speed:

$$
u_{i, J}=u_{i, J}^{*}+\left(p_{I-1, J}^{\prime}-p_{I, J}^{\prime}\right) d_{i, J}
$$

\section{The Calculation Results and Analysis}

As an example, the fluid flow through orthogonal surface is calculated by the method presented in this article, where $\mathrm{u}=\mathrm{Ax}, \mathrm{V}=-\mathrm{AY}$, Tin=500K, Tw=100K.Model size is shown in Fig. 2:

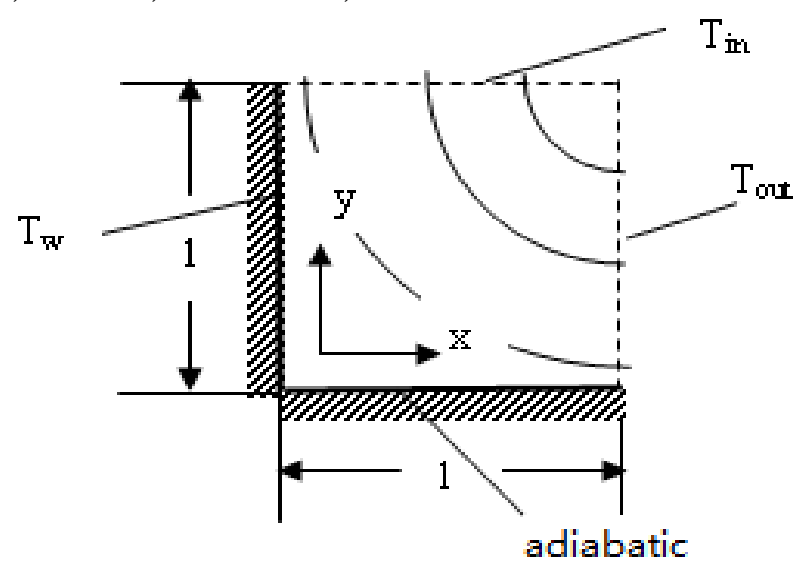

Fig.2 Fluid flow through orthogonal surface

The bottom boundary is adiabatic, staggered grid was used in $\mathrm{x}$ direction, and collocated grid was used in y direction. The calculation results is shown in Figure 4. 


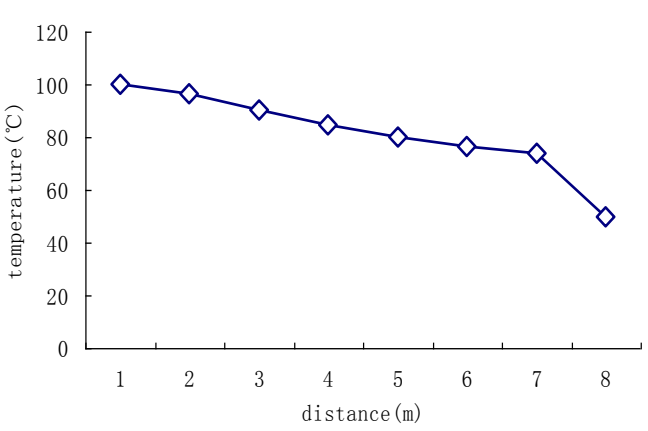

$\mathrm{y}=0.125 \mathrm{~m}$

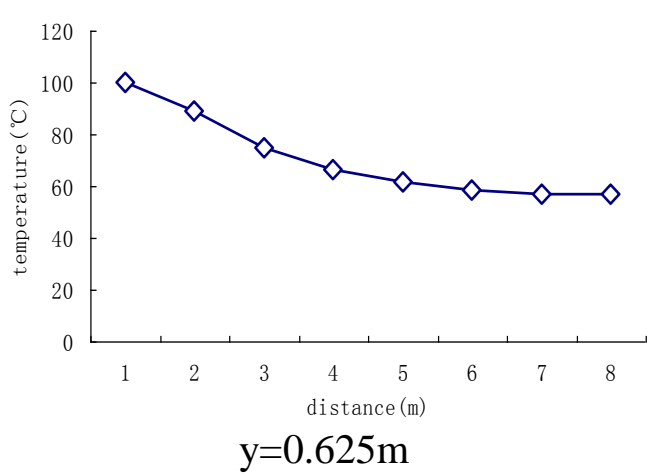

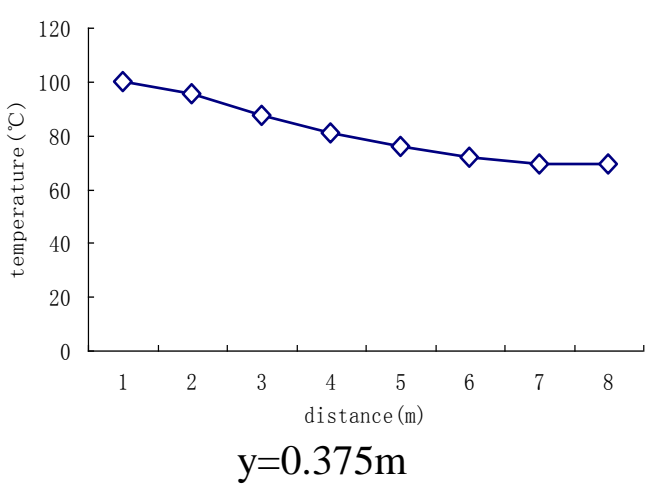

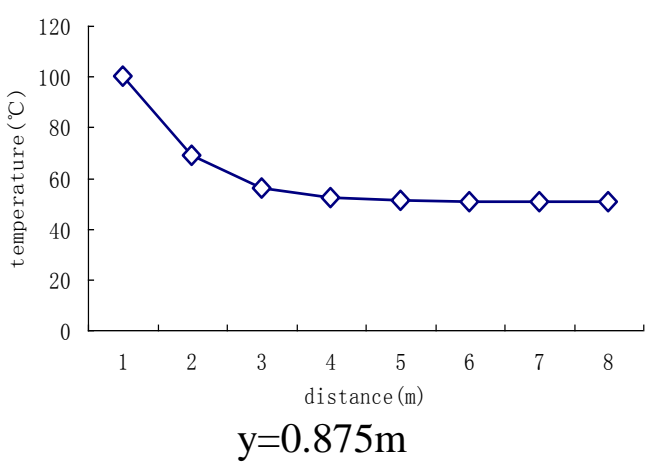

Fig.3 Temperature distribution curve of rectangular planar flow field by single staggered grid algorithm

From Fig. 3, it could be concluded that the temperature of the flow gradually reduced along the $\mathrm{x}$ direction, and the highest temperature is $100^{\circ} \mathrm{C}$ at the left side of the flow field. The largest temperature difference between the two ends of the flow field is about $50^{\circ} \mathrm{C}$ at $\mathrm{y}=0.125 \mathrm{~m}$. While the minimum temperature difference is about $30^{\circ} \mathrm{C}$ at $\mathrm{y}=0.375 \mathrm{~m}$. The lowest temperature is about $50^{\circ} \mathrm{C}$ located in the export of the flow field. In order to verify the accuracy of the results, the problem was calculated by SIMPLE algorithm based on traditional staggered grid system, the results are shown in Fig. 4:

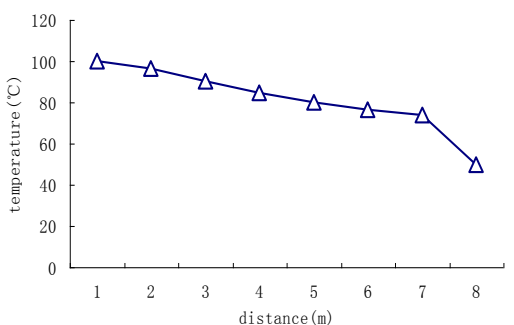

$\mathrm{y}=0.125 \mathrm{~m}$

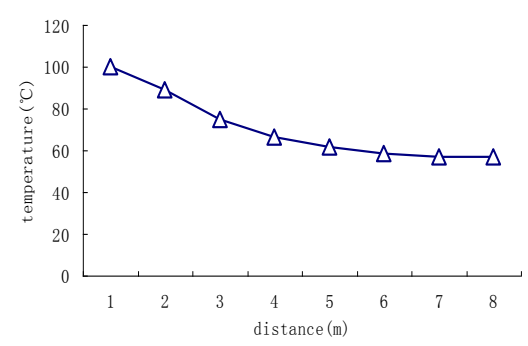

$\mathrm{y}=0.625 \mathrm{~m}$

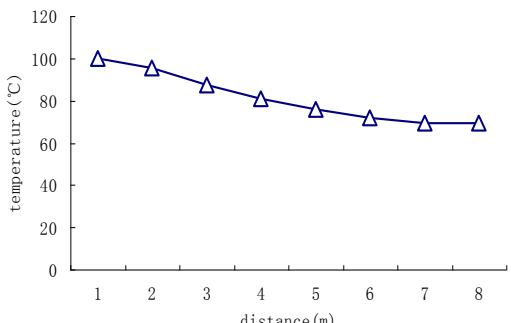

$\mathrm{y}=0.375 \mathrm{~m}$

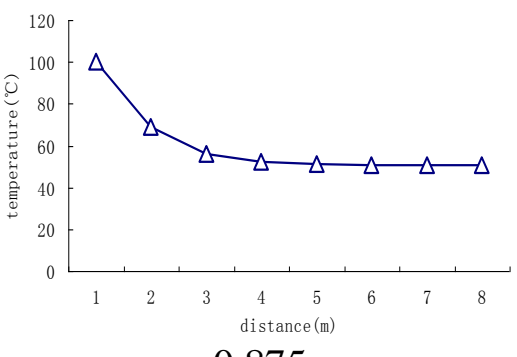

$\mathrm{y}=0.875 \mathrm{~m}$

Fig.4 Temperature distribution curve of rectangular planar flow field by traditional staggered grid algorithm 
After comparing Fig. 3 to Fig. 4, it could be concluded that the results of single direction staggered grid are in good agreement with that of traditional staggered grid. Compared with the staggered grid system, single direction staggered grid reduces a set of grid for two-dimensional fluid flow problems, which can effectively reduce the number of variables and has better grid adaptability. So the single direction staggered SIMPLE algorithm has certain engineering application value.

\section{Conclusion}

SIMPLE algorithm based on single direction staggered grid system was used in this article to calculate the Navier-Stokes equations. The influence on numerical calculation by unreasonable pressure field were inhibited by the momentum interpolation. The speed and pressure correction equations of different control volumes were derived.

As an example, the flow and heat conduction of fluid through the right-angle plane in sewage treatment system were calculated in this article. After comparing Figure 4 to Figure 5, it can be concluded that the calculated results of single direction staggered grid are in good agreement with that of traditional staggered grid. The single direction algorithm presented in this article has less variables than traditional staggered grid, and simpler grid system, which has good application value in engineering problems.

\section{Acknowledgments}

This article is supported by the National Science Foundation of China (no. 11202228).

\section{References}

[1] ZHOU Zhu-sheng, LI Yuan. High order staggered grids non stationary phase shift method post stack depth migration. PROGRESS IN GEOPHYSICS, Vol. 28, (2013), p. 658

[2] WANG Li-ye, OUYANG Jie, ZHAO Zhi-feng, etc. Simulation and Comparison of FVMs Based on Non-staggered and Staggered Grid for Planar Contraction Flows. CHINESE JOURNAL OF ENGINNERING MATHEMATICS, Vol. 26, (2006), p. 85

[3] WANG Ru-yun, QIU Jian-xian, DAI Jia-zun, etc. A high resolution Gauss scheme with staggered grid for shallow water equation. ADVANCES IN WATER SCIENCE, Vol. 13, (2002), p. 403

[4] Acharya S, Moukalled F. Improvement to incompressible flow calculation on a nonstaggered curvilinear grid. Numerical Heat Transfer, Part B, Vol.15, (1989), p. 131

[5] YE Ping, ZHANG Jing-zhou. SIMPLE Algorithm for solution of Navier-Stokes Equations on a Semi-staggered Grid. Journal of Nanjing University of Aeronautics \& Astronautics. Vol. 36, (2004), p. 11

[6] FU Xiao-yan, GAO Ge. Study of SIMPLE algorithm for compressible flows on non-staggered grids. Journal of Aerospace Power, vol. 22, (2007), p. 1673

[7] LIU Yilun, LIU Weitao, Li Songbo. THE FLOW AND INTERNAL LEAKAGE OF ANNULAR SPIRAL FLOW IN HYDRAULIC ROTARY ACTUATOR. Mechanics and Practice, vol. 32, (2010), p. 27

[8] BAI Yu-guang, TUO Chao-zhi, SUN Dong-ke, etc. Gust response analysis of the composite wing with pseudo excitation method and CFD. Chinese Journal of Computational Mechanics, vol. 29, (2012), p. 556

[9] HAO Wen-lan, CHEN Bao-ming, CHI Guang-zhou, etc. Numerical Simulation of the Driven Cavity FLow. JOURNAL OF ENGINEERING THERMOPHYSICS, vol. 33, (2012), p. 295 
[10] YANG Zhong, DU Jian-yi, Xu Jian-zhong. TURBULENCE MODEL BASED NUMERICAL INVESTIGATION OF TRANSITIONAL FLOWS. JOURNAL OF ENGINEERING THERMOPHYSICS, Vol. 2, (2010), p. 231

[11]HUANG Guoxian, ZHOU Jianjun, CHEN Jieren. Convergence of flow models using staggered and collocated grids with complicated boundaries. J Tsinghua Univ(Sci \& Tech), vol. 46, (2006), p. 785

[12]ZHANG Jing-zhou, JI Hong-Hu, WANG Suo-Fang. NUMERRICAL STUDY FOR TURBULENT FLOW AND HEAT TRANSFER INSIDE ROTOR-STATOR DISC CAVITY WITH RADIAL OUTFLOW[J]. JOURNAL OF ENGINEERING THERMOPHYSICS, Vol. 22, (2001), p. 121 